\title{
Spin-wave dynamics in the helimagnet FeGe studied by small-angle neutron scattering
}

\author{
S.-A. Siegfried, ${ }^{1}$ A. S. Sukhanov, ${ }^{2,3, *}$ E. V. Altynbaev, ${ }^{2,3}$ D. Honecker, ${ }^{4}$ A. Heinemann, ${ }^{1}$ \\ A. V. Tsvyashchenko, ${ }^{5}$ and S. V. Grigoriev ${ }^{2,3}$ \\ ${ }^{1}$ German Engineering Materials Science Centre (GEMS) at Heinz Maier-Leibnitz Zentrum (MLZ), \\ Helmholtz-Zentrum Geesthacht GmbH, Lichtenbergstrasse 1, 85747 Garching bei München, Germany \\ ${ }^{2}$ Petersburg Nuclear Physics Institute, Gatchina, St-Petersburg 188300, Russia \\ ${ }^{3}$ Faculty of Physics, Saint-Petersburg State University, Ulyanovskaya 1, Saint-Petersburg 198504, Russia \\ ${ }^{4}$ Institute Laue Langevin, Grenoble, 38042 Grenoble, Cedex 9, France \\ ${ }^{5}$ Institute for High Pressure Physics, Russian Academy of Sciences, 142190 Troitsk, Moscow, Russia \\ (Received 27 September 2016; revised manuscript received 7 February 2017; published 12 April 2017)
}

\begin{abstract}
We have studied the spin-wave stiffness of the Dzyaloshinskii-Moriya helimagnet FeGe in a temperature range from $225 \mathrm{~K}$ up to $T_{C} \approx 278.7 \mathrm{~K}$ by small-angle neutron scattering. The method we have used is based on [Grigoriev et al., Phys. Rev. B 92, 220415(R) (2015)] and was extended here for the application in polycrystalline samples. We confirm the validity of the anisotropic spin-wave dispersion for FeGe caused by the Dzyaloshinskii-Moriya interaction. We have shown that the spin-wave stiffness $A$ for the FeGe helimagnet decreases with a temperature as $A(T)=194\left[1-0.7\left(T / T_{C}\right)^{4.2}\right] \mathrm{meV} \AA^{2}$. The finite value of the spin-wave stiffness $A=58 \mathrm{meV} \AA^{2}$ at $T_{C}$ classifies the order-disorder phase transition in FeGe as being the first-order one.
\end{abstract}

DOI: 10.1103/PhysRevB.95.134415

\section{INTRODUCTION}

The cubic B20 compounds have a noncentrosymmetric crystal structure described by the $P 2{ }_{1} 3$ space group. The lack of a symmetry center of the crystal structure produces the chiral spin-spin Dzyaloshinskii-Moriya (DM) interaction $[1,2]$. According to the model by Bak and Jensen (BJ) [3] and Nakanishia et al. [4], the major ferromagnetic exchange interaction $J$ together with the DM interaction $D$ produces a (homochiral) structure in these systems below $T_{C}$. The energy landscape in these systems is given by $J$ and $D$, which are balanced via the helix wave-vector $k_{s}=D / J$. The anisotropic exchange interaction has been added to the model, changing slightly the value and fixing the direction of the wave-vector $\boldsymbol{k}_{s}$ along the principal cubic axis. As noticed by Nakanishia and co-workers [4] and Grigoriev and co-workers [5], the cubic anisotropy can play an important role in the case of relatively small values of the helix wave-vector $k_{s}$. If the anisotropic energy gets comparable to the DM interaction, it can destabilize the entire helix structure and stabilizes instead the ferromagnetic state.

The external magnetic-field $H_{c 1}$ is needed to rotate the helix wave-vector $\boldsymbol{k}_{s}$ towards the field direction and, therefore, it is a measure for the anisotropy of the system. According to Ref. [6], the energy difference between the helical and the collinear full-polarized (FP) state can be directly measured by the second critical field $H_{c 2}$. This energy is equal to $g \mu_{B} H_{c 2} \approx A k_{s}^{2}$, where $A=J S a^{2}$ is the spin-wave (SW) stiffness, $S$ is the ordered spin, and $a$ is the lattice constant. The experimental parameters $k_{s}, H_{c 1}, H_{c 2}$, and $S$ describe completely the magnetic system of such compounds.

The compound FeGe shows a significant difference in the parameters of the magnetic structure compared to the other B20 helimagnet $\mathrm{MnSi}[7,8]$. The helix pitch length is nearly

\footnotetext{
*Currently at the Max-Planck Institute for Chemical Physics of Solids, D-01187 Dresden, Germany.
}

four times larger and equal to $\lambda_{h} \approx 700 \AA$. The ordering temperature $T_{C} \approx 278.7 \mathrm{~K}$ is nearly ten times higher than the $T_{C}$ for MnSi. As for the anisotropic interactions the helix wave vector is pinned along the [111] direction in $\mathrm{MnSi}$ at all temperatures below $T_{C} \approx 29.5 \mathrm{~K}$, whereas the helix wave vector in FeGe is pinned along the [100] direction in the high-temperature range between $T_{C}=278.7 \mathrm{~K}$ and $T_{2 \downarrow}=$ $211 \mathrm{~K} / T_{2 \uparrow}=245 \mathrm{~K}$ and rotates towards the [111] direction in the low-temperature part of the ordered phase for $T<T_{2 \downarrow} \uparrow$. According to the Bak and Jensen model a temperaturedriven rotation of the spiral from [100] to [111] goes along with a change in the sign of the second-order gradient in the free-energy expansion [3]. Nevertheless, magnetization measurements indicated that the rotation of the helix axis at $T_{2 \downarrow, \uparrow}$ can be explained by an interplay of constants of the fourth and sixth terms of the cubic anisotropy [9].

Although the spin-wave dynamics of MnSi has been studied intensively in the past [10-16], it was never the case for FeGe due to the lack of a sufficient large amount of single-crystalline samples, which is necessary for the triple-axis spectroscopy. The recently proposed method [16] to determine the spin-wave stiffness in the helical magnets based on DM interaction in the FP state, using polarized small-angle neutron scattering (SANS), can be extended to be used for the polycrystalline FeGe compounds. This method originally was developed and applied to measure the spin-wave stiffness in ferromagnetic compounds [17-21]. The presence of the DM chiral interaction leads to the chirality of the spin waves in the FP state of helimagnets. This fact is related to the completely anisotropic dispersion relation of magnons, which reads

$$
\epsilon_{\boldsymbol{q}}=A\left(\boldsymbol{q}-\boldsymbol{k}_{s}\right)^{2}+H-H_{c 2}
$$

for the magnetic field above the critical value of $H_{c 2}$ [22]. It can be shown analytically that the inelastic scattering of the neutrons in this case is concentrated mostly around the momentum transfers corresponding to $\pm \boldsymbol{k}_{s}$ within two narrow cones limited by the cutoff angle $\theta_{C}$ for the energy gain/energy 
loss, respectively [16],

$$
\theta_{C}^{2}(H)=\theta_{0}^{2}-\frac{\theta_{0}}{E_{i}} H+\theta_{B}^{2},
$$

where $\theta_{B}$ is the Bragg angle of the scattering on the spin spiral with the length $2 \pi / k_{s}$ and $E_{i}$ denotes the energy of the incident neutrons. The cutoff angle is connected to the spin-wave stiffness via the dimensionless parameter $\theta_{0}=\left(2 A m_{n}\right)^{-1}$, where $m_{n}$ is the neutron mass. Here we assume conditions $\omega \ll E_{i}$ and $\omega \ll T$ to be fulfilled in the limit of small-angle neutron scattering. The first condition allows one to split momentum transfer into an elastic component perpendicular to $\boldsymbol{k}_{i}$ and an inelastic one parallel to $\boldsymbol{k}_{i}$. The Bose factor $[1-\exp (-\omega / T)]^{-1}$ can be replaced by $T / \omega$ accounting for the second condition. Despite being $\omega$ odd, the cross section of the inelastic scattering being integrated over the energy transfer contains the polarization-dependent part due to the peculiarity of the aforementioned asymmetric dispersion relation. As was demonstrated in Ref. [16], one can distinguish the scattering from the helimagnons in a homochiral crystalline sample using polarized neutrons in the SANS experiment.

In this paper we show the possibility to measure the SW stiffness in the polycrystalline samples using nonpolarized neutrons. As the sample contains both left and right crystallites, it is, therefore, not possible to demonstrate the chiral nature of the spin-wave scattering. Nevertheless, it is possible to detect two circles on a scattering map with radius $\theta_{C}$ and centered at the Bragg peak $\theta_{B}$. The spin-wave stiffness $A$ is measured in the temperature range below $T_{C}$ by finding the cutoff angle in accordance to Eq. (2).

This paper is organized in the following way: Secs. II and III give the results of the small-angle neutron-scattering measurements of the FeGe compound. Section IV presents the conclusions.

\section{ELASTIC SMALL-ANGLE NEUTRON MEASUREMENTS}

The SANS experiment was performed at the D11 instrument at the Institut Laue Langevin (Grenoble, France). An unpolarized beam with a mean wavelength of $\lambda=0.6 \mathrm{~nm}$ was used. A magnetic field $(0.0-0.5 \mathrm{~T})$ was applied perpendicular to the incident beam. The FeGe sample with the mass of 0.1 $\mathrm{g}$ was the same as used in our previous work [23]. It was synthesized using the high-pressure method (see Ref. [24] for details).

A typical SANS map for $T=250 \mathrm{~K}$ and $H \gtrsim H_{c 1}=$ $0.075 \mathrm{~T}$ is shown in Fig. 1(a). Several Bragg peaks appear on the left and right sides of the scattering map where the center of the map corresponds to $Q_{x}=0, Q_{y}=0$. The two peaks closest to the center are from the helical structure at $Q= \pm 0.09 \mathrm{~nm}^{-1}$. The others are clearly the higher orders of multiple scattering. The spiral wave vector is aligned to the direction of the magnetic field.

Figure 1(b) represents the integrated intensities of the Bragg peak $I$ at $Q= \pm 0.09 \mathrm{~nm}^{-1}$ as a function of the field for different temperatures between 5 and $T_{C} \approx 278.7 \mathrm{~K}$. The sample has been cooled down from $T_{C}$. The cooling has been stopped at 16 different temperatures in-between, and a field scan with an increasing magnetic field was performed at each
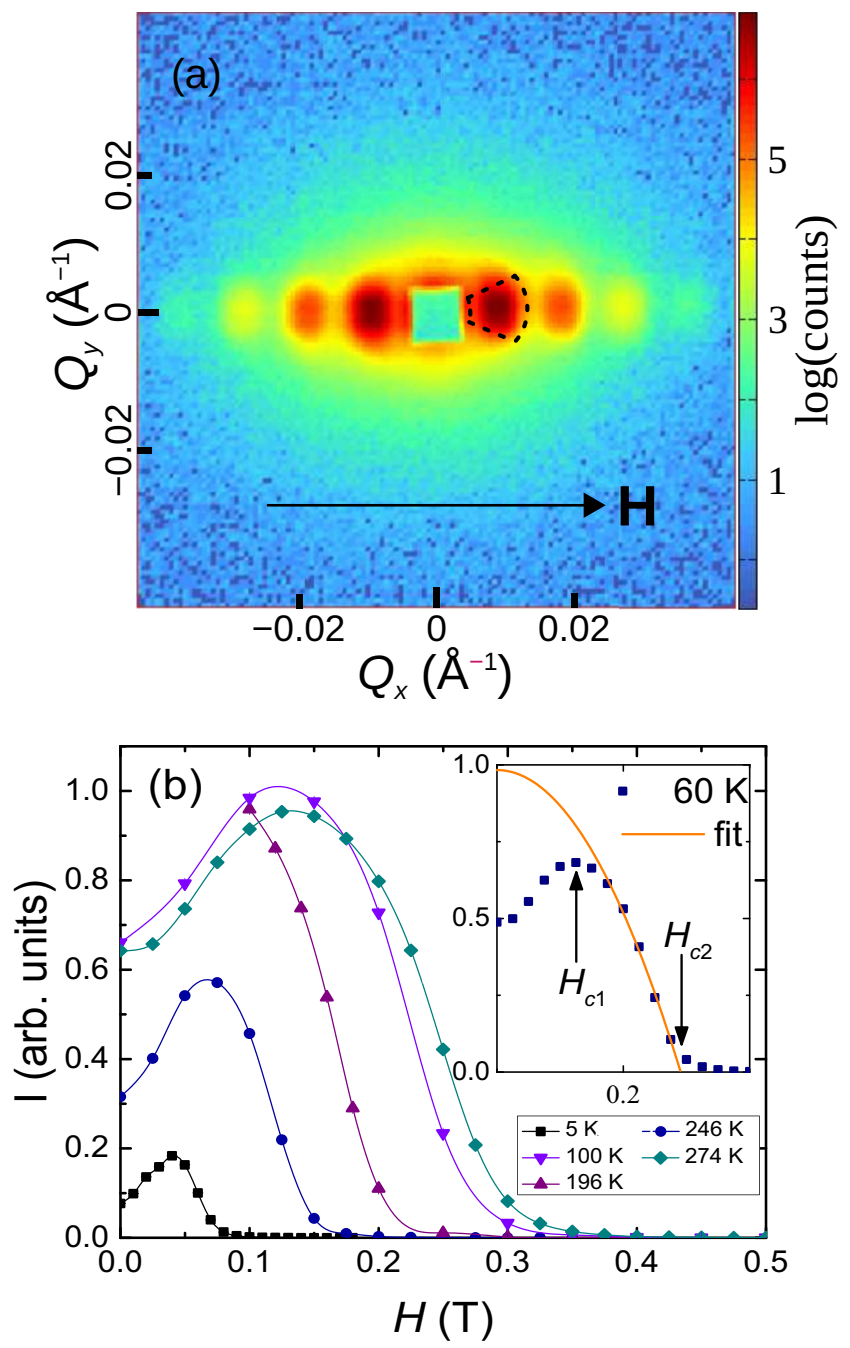

FIG. 1. (a) Typical SANS scattering pattern from a monodomain helix structure below $H_{c 2}, T=250 \mathrm{~K}$ and a magnetic-field $H=$ $0.075 \mathrm{~T}$. (b) Integral intensity of the marked Bragg peak against the magnetic field at different temperatures between 5 and $274 \mathrm{~K}$. The solid lines are guides for the eyes. The inset shows an illustration of the determination of $H_{c 2}$ at $60 \mathrm{~K}$. The BJ model is used to provide the fitting function (see the text for more details).

temperature. As is shown in the inset, the first critical field $H_{c 1}$ is defined as a point of the maximal intensity, and this is the point where all spirals are aligned along the field. The difference from the previously used method [23] for $\mathrm{Fe}_{1-x} \mathrm{Co}_{x} \mathrm{Ge}$ is worth mentioning where we determined $H_{c 1}$ as the starting point for the increasing intensity, corresponding to the spiral starts aligning along the field direction. The second critical field $H_{c 2}$ is determined from the zero point of the fitting function according to the Bak-Jensen model. The model predicts that the cone angle $\alpha$, counted from the helix plane, increases with the field as [6]

$$
\sin \alpha=\frac{H}{H_{c 2}} .
$$

Thus, the intensity of elastic scattering subsides $I \sim \cos ^{2} \alpha \sim$ $1-\left(H / H_{c 2}\right)^{2}$. However, a tail of the intensity can still be observed above $H_{c 2}$ [Fig. 1(b)]. This tail is better pronounced 


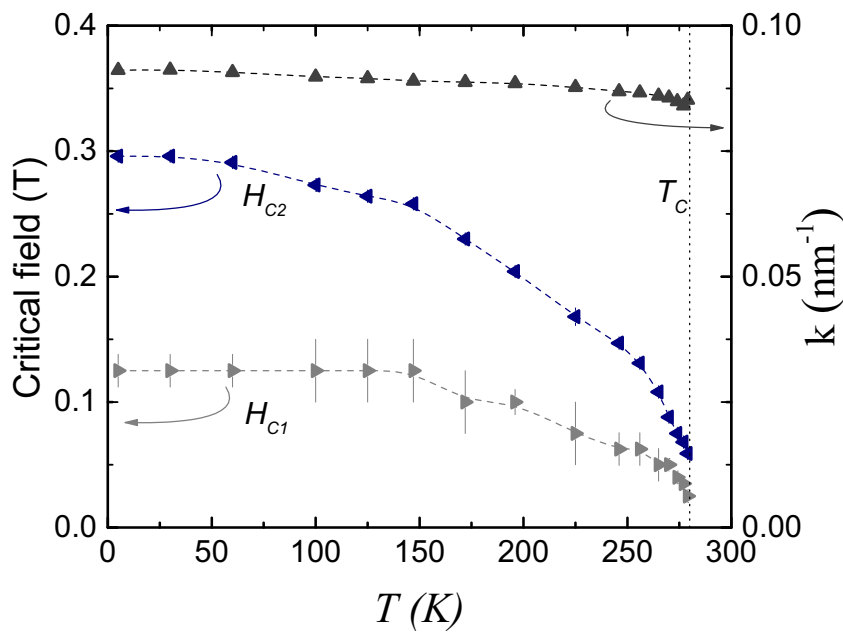

FIG. 2. The temperature dependence of the value of the helix wave-vector $k_{s}$ and both critical fields $H_{c 1,2}$. The dashed lines are guides for the eyes.

at the low temperatures, whereas it becomes invisible in the high-temperature region. The phenomenon may be caused by the influence of the cubic anisotropy that makes the critical field $H_{c 2}$ depending on the orientation of the applied field with respect to the principal crystallographic axes [5]. The magnetic structure in the randomly oriented crystallites undergoes the phase transition to the field polarized state at different strengths of the field from the minimal $H_{c 2}=A k_{s}^{2}-8 G /(3 S)$ to the maximal value of $H_{c 2}=A k_{s}^{2}+4 G / S$, where $G$ is the constant of the cubic anisotropy.

The helix wave-vector $\left|\boldsymbol{k}_{s}\right|$ has been determined as a center of the Gaussian function fitting the helical Bragg peak in a zero magnetic field for each temperature. The temperature dependence of the helix wave vector is shown in Fig. 2, and it is nearly constant and equal to $0.09 \mathrm{~nm}^{-1}$ in the whole temperature range. Furthermore, the $H-T$ phase diagram for FeGe between $5 \mathrm{~K}$ and $T_{C}$ is shown in Fig. 2. The second critical field $H_{c 2}$ decreases slowly from a maximum value of $0.3 \mathrm{~T}$ at low temperatures and tends to zero at $T_{C}$. The first critical field $H_{c 1}$ stays roughly constant at $0.1 \mathrm{~T}$ between 5 and $180 \mathrm{~K}$ and decreases with further temperature increases towards zero at $T_{C}$. As was mentioned, $H_{c 2}$ is the measure of the difference in energies of the spiral state and the FP state and is equal to $A k_{s}^{2}$. As far as $k_{s}$ shows no change, a temperature decrease in $H_{c 2}$ is expected to be driven by a decrease in $A$ and is related to the softening of the magnetic structure with the temperature. Meanwhile the mechanism standing behind the temperature changing of $H_{c 1}$ remains less clear.

\section{SMALL-ANGLE NEUTRON MEASUREMENTS OF THE SPIN-WAVE STIFFNESS}

The inset of Fig. 3 shows a typical SANS map for FeGe, which is taken above $H_{c 2}$. The position of the detector optimally was set to study the scattering of the spin waves. Due to the limited detector area one may lose the cutoff angle adjusting the position of the Bragg peak further away from the direct beam. As the field exceeds $H_{c 2}$, the elastic scattering disappears, and only the inelastic scattering centered

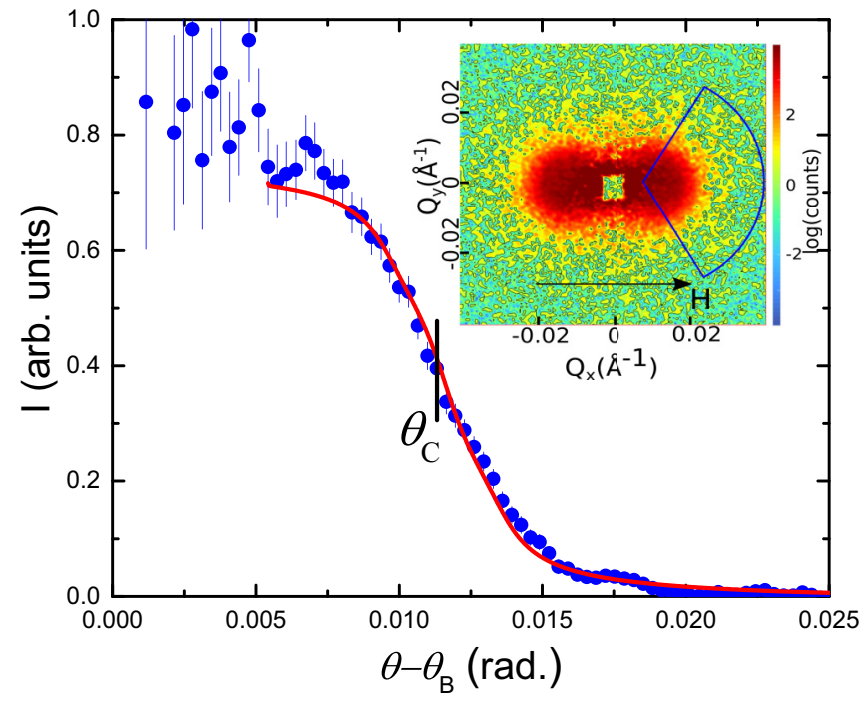

FIG. 3. The averaged intensity profile at $T=250 \mathrm{~K}$ and $H=$ $0.3 \mathrm{~T}$. The sigmoid function fits the steplike part of the scattering. The cutoff angle is shown. The inset: SANS map above the critical field $T=250 \mathrm{~K}$ and $H=0.3 \mathrm{~T}$.

at $\boldsymbol{Q}= \pm \boldsymbol{k}_{s}$ remains [16]. This scattering consists of the strong diffuse scattering in the vicinity of the former Bragg peak and a round spot limited by the critical angle $\theta_{C}$. The diffuse scattering at $\boldsymbol{Q}= \pm \boldsymbol{k}_{s}$ is maximal at $H \approx H_{c 2}$ and strongly suppressed by an increase in the field. According to Eq. (2) the spin-wave part of the scattering becomes narrower with a further increase in the field and has vanished at a certain $H_{o f f}$ above $H_{c 2}$. Using Eq. (1), we define this value as

$$
\theta_{0}^{2}-\frac{\theta_{0}}{E_{i}} H_{\mathrm{off}}=0
$$

and obtain $H_{\text {off }}=\theta_{0} E_{i}$. To define the cutoff angle $\theta_{C}$, a measurement of the background intensity at $H>H_{\text {off }}$ was subtracted from the other scattering maps. To improve the statistics, the scattering intensity was azimuthally averaged over the angular sector of $120^{\circ}$ with the center positioned at $\boldsymbol{Q}= \pm \boldsymbol{k}_{s}$ as shown in Fig. 3. The resultant curve is shown in Fig. 3 for $T=250 \mathrm{~K}$ and $H=0.25 \mathrm{~T}$. From the analysis of the $I$ versus $\theta-\theta_{B}$ plot the cutoff angle can be extracted.

Nevertheless, a sharp cutoff of the intensity was not observed due to the damping of spin waves and/or the $Q$ resolution of the SANS instrument, which is equal to 0.001 rad. The expected steplike intensity profile is smeared into the smoothly decreasing curve. This smeared steplike edge of the measured intensity was fitted by the following sigmoid function, which captures the main features of the scattering:

$$
I(\theta)=I_{0}\left\{\frac{1}{2}-\left(\frac{1}{\pi} \arctan \left[\frac{2\left(\theta-\theta_{C}\right)}{\delta}\right]\right)\right\} .
$$

The position of the cutoff angle was determined as the center of the arctan function $\theta_{C}$. Its width $\delta$ is related to the spin-wave damping $\Gamma$ and can roughly be estimated as $\Gamma=\delta E_{n}$.

Figure 4 shows the intensities as function of the angle $\theta-\theta_{B}$ at different values of the magnetic field for $T=250 \mathrm{~K}$ together with the corresponding fitting curves. The curves show a decrease in the value of the cutoff angle together with a strong 


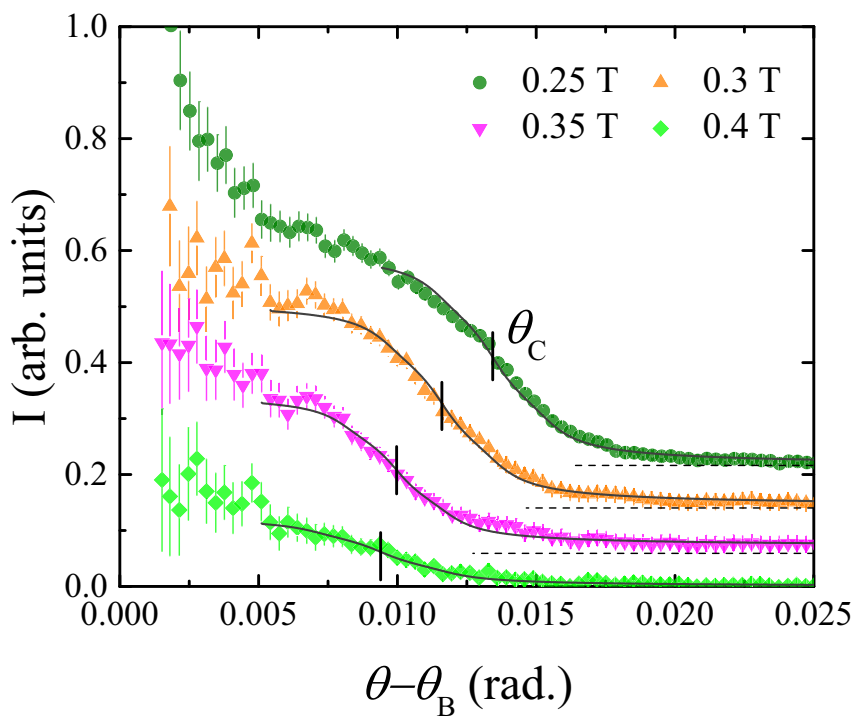

FIG. 4. The azimuthally averaged intensities at $T=250 \mathrm{~K}$ for different magnetic fields between $H=0.25$ and $H=0.4 \mathrm{~T}$. The fitting functions are chosen analogously to Fig. 3. The curves are shifted by a constant with respect to each other for clarity.

suppression of the scattering $\theta \approx \theta_{B}$ with an increase in the field. The latter is related to the gap in the spin-wave spectrum increasing with the field when the quasielastic scattering with relatively low $\omega$ is forbidden. Consequently, the intensity being proportional to $1 /\left(H-H_{c 2}\right)$ decreases at $\left(\theta \sim \theta_{B}\right)$.

The intensity for $H=0.35 \mathrm{~T}$ is plotted in Fig. 5(a) for different temperatures between 196 and $270 \mathrm{~K}$ including fitting functions. Two parameters $\theta_{C}$ and $\delta$ have been extracted from the fitting procedure. Figure 5(c) shows the field dependence of the damping related parameter $\delta$ for different temperatures between 246 and $277 \mathrm{~K}$, which is nearly constant for each temperature. Figure 5(b) shows the temperature dependence of $\delta, \delta$ increases drastically close to $T_{C}$ in accordance with theoretical expectations. The evaluation in accordance with $\Gamma=\delta E_{i}$ gives the values changing from 20 to $250 \mu \mathrm{eV}$ for the temperature range from 240 to $280 \mathrm{~K}$. The linear dependence of the squared cutoff angle $\theta_{C}^{2}$ against the magnetic field is shown in Fig. 6 for $T=246,265$, and $274 \mathrm{~K}$. We were not able to determine the cutoff angle for the temperatures below $225 \mathrm{~K}$ because of the intensive quasielastic scattering arising in the position of the former Bragg peak position.

The square of the cutoff angle depends linearly on the field in accordance with Eq. (2). With the help of Eq. (2) one can determine the value of the parameter $\theta_{0}$ and determine the spinwave stiffness with high accuracy. The spin-wave stiffness, obtained from the cutoff angle for different temperatures is shown in Fig. 7. The measured temperature dependence was fitted by the power law: $A(T)=a\left[1-c\left(T / T_{C}\right)^{z}\right]$, and the parameters are found to be $a=(0.194 \pm 0.010) \mathrm{eV} \AA^{2}, c=$ $0.70 \pm 0.01$, and $z=4.20 \pm 0.48$. The value of the stiffness $A$ does not tend to 0 as one would expect at $T_{C}$ for the second-order phase transitions but is finite at $A\left(T_{C}\right)=a \times$ $0.3=0.058 \mathrm{eV} \AA^{2}$. This fact clearly classifies the magnetic phase transition in FeGe as being of the first order. This is not so surprising as for the archetypal system, namely, for $\mathrm{MnSi}$,
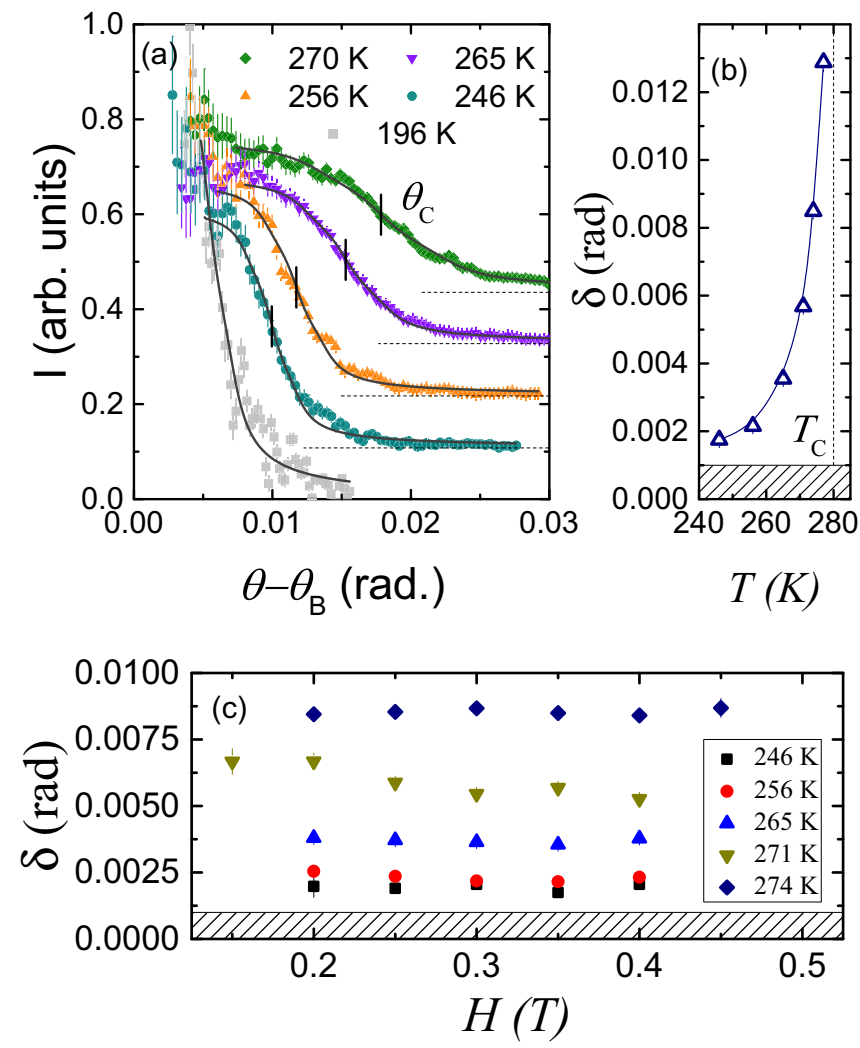

FIG. 5. (a) The temperature evolution of the intensity profiles from $T=196$ to $T=270 \mathrm{~K}$ at $H=0.35 \mathrm{~T}$. The curves are shifted by a constant with respect to each other for clarity. (b) The relative change in width of the steplike part $\delta$ for $H=0.35$. (c) The relative change in width of the steplike part $\delta$ for $T=246-277 \mathrm{~K}$ between $H=0.15$ and $0.45 \mathrm{~T}$. The instrumental resolution is marked by the patterned area.

it is experimentally well established that the helimagnetic to paramagnetic transition is first order [25-28]. It was also theoretically predicted that the helimagnetic ordering in the systems with DM interaction should universally result in a first-order transition (the so-called Brazovskii scenario) [28]. However the recent magnetization and thermal studies on FeGe have suggested that the transition should be second order [29].

Nevertheless, since FeGe is a helimagnet, the magnetization does not represent the order parameter, and therefore the kind

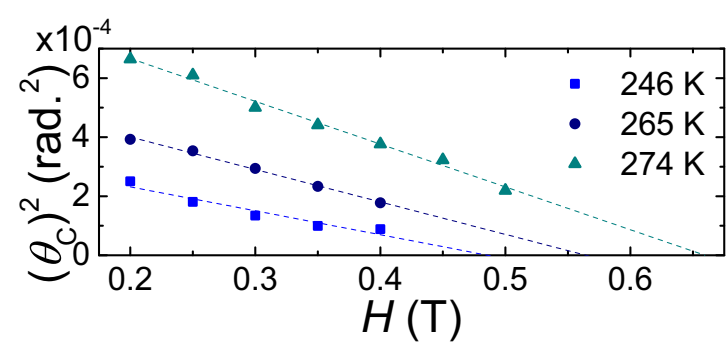

FIG. 6. The field dependence of the square of the cutoff angle $\theta_{C}^{2}$ at $T=246,265$, and $274 \mathrm{~K}$. The intersections between the linear fits and the $H$ axis determine $H_{\text {off }}$. 


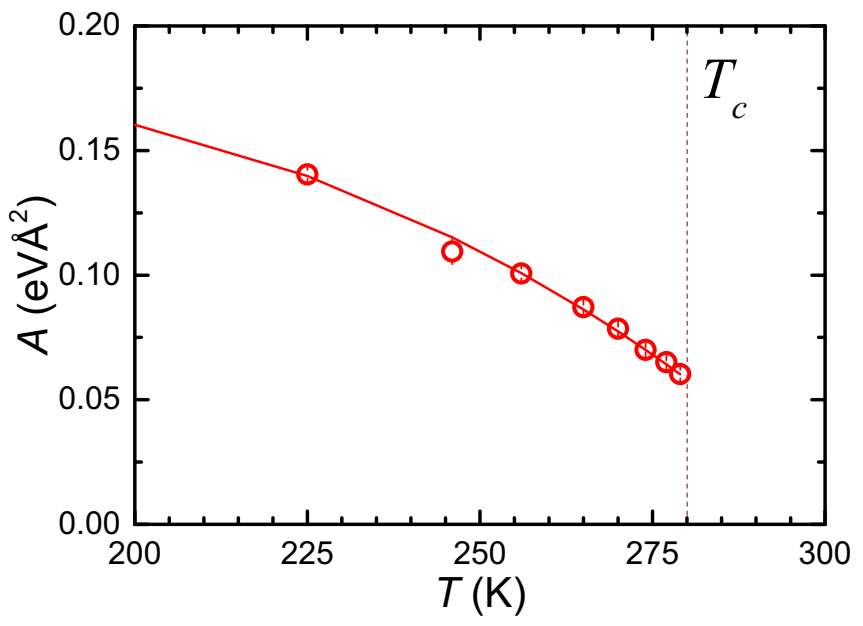

FIG. 7. Temperature dependence of the spin-wave stiffness measured by the cutoff angle with the corresponding fit.

of order transition can be hard to differentiate from specific heat measurements at high temperatures where the phonon background is large. Our experimental findings contradict those published on FeGe [29] but support the theoretical experimental understanding of the DMI compounds related to the archetypal MnSi compound [28].

It is interesting to note that the spin-wave stiffness can also be estimated from the theory by Bak and Jensen [6] using the ratio relating the critical magnetic-field $H_{c 2}$ and the difference in the energies between the FP and the helical states $g \mu_{B} H_{c 2}=$ $A k_{s}$. This relation has been confirmed experimentally to be valid for $\mathrm{MnSi}$ in the whole temperature range below $T_{C}$ [16]. However, the use of this relation is limited as the expression should be corrected taking into account the cubic anisotropy [5]. It is especially important for the compounds with extremely small $k_{s}$, which is the case for FeGe. Additional uncertainty can come from the determination of the critical field $H_{c 2}$ caused by the demagnetization effect. We estimate that the values of the critical fields given in Fig. 2 are a factor of 1.5 larger than real numbers. Due to the higher magnetic moment and the polycrystalline nature of the used FeGe samples, this effect might play a bigger role for FeGe than for the previous investigated single-crystalline MnSi [16].

\section{CONCLUSION}

In conclusion, we have experimentally determined the spin-wave stiffness in the high-temperature phase of the FeGe compound. We confirm the validity of the spin-wave dispersion relation for another helimagnet with DM interaction [Eq. (1)]. Particularly, the experiment had shown that the minimum of the dispersion curve is shifted along the field axis from the position $q=0$ to the value of $\boldsymbol{k}_{s}$. Furthermore, we demonstrated the ability of small-angle neutron scattering to measure the spinwave stiffness in polycrystalline samples of DM helimagnets in the full-polarized state with acceptable statistics in reasonable time. The method allows the determination of the spin-wave stiffness in a broad temperature range and above all opens up completely new possibilities in the investigation of the parameter of the spin-wave stiffness in other representatives of DM helimagnets, which could be synthesized as powder only.

\section{ACKNOWLEDGMENTS}

We thank Professor S. V. Maleyev for valuable discussions and Professor A. Schreyer for continuous interest and support. The work was supported by the Russian Foundation of Basic Research (Grants No. 14-22-01073 and No. 14-02-00001) and the special program of the Department of Physical Science, Russian Academy of Sciences.
[1] I. Dzyaloshinsky, J. Phys. Chem. Solids 4, 241 (1958).

[2] T. Moriya, Phys. Rev. 120, 91 (1960).

[3] P. Bak and M. H. Jensen, J. Phys. C 13, L881 (1980).

[4] O. Nakanishia, A. Yanasa, A. Hasegawa, and M. Kataoka, Solid State Commun. 35, 995 (1980).

[5] S. V. Grigoriev, A. S. Sukhanov, and S. V. Maleyev, Phys. Rev. B 91, 224429 (2015).

[6] S. V. Maleyev, Phys. Rev. B 73, 174402 (2006).

[7] B. Lebecht, J. Bernhard, and T. Freltloft, J. Phys.: Condens. Matter 1, 6105 (1989).

[8] Y. Ishikawa, K. Tajima, D. Bloch, and M. Roth, Solid State Commun. 19, 525 (1976).

[9] L. Lundgren, O. Beckman, V. Attia, S. P. Bhattacherjee, and M. Richardson, Phys. Scr. 1, 69 (1970).

[10] Y. Ishikawa, G. Shirane, J. A. Tarvin, and M. Kohgi, Phys. Rev. B 16, 4956 (1977).

[11] J. A. Tarvin, G. Shirane, Y. Endoh, and Y. Ishikawa, Phys. Rev. B 18, 4815 (1978).

[12] F. Semadeni, P. Boni, Y. Endoh, B. Roessli, and G. Shirane, Physica B 267, 248 (1999).
[13] M. Janoschek, F. Bernlochner, S. Dunsiger, C. Pfleiderer, P. Boni, B. Roessli, P. Link, and A. Rosch, Phys. Rev. B 81, 214436 (2010).

[14] M. Kugler, G. Brandl, J. Waizner, M. Janoschek, R. Georgii, A. Bauer, K. Seemann, A. Rosch, C. Pfleiderer, P. Boni, and M. Garst, Phys. Rev. Lett. 115, 097203 (2015).

[15] T. Schwarze, J. Waizner, M. Garst, A. Bauer, I. Stasinopoulos, H. Berger, C. Pfleidererand, and D. Grundler, Nat. Mater. 14, 478 (2015).

[16] S. V. Grigoriev, A. S. Sukhanov, E. V. Altynbaev, S.-A. Siegfried, A. Heinemann, P. Kizhe, and S. V. Maleyev, Phys. Rev. B 92, 220415(R) (2015).

[17] A. I. Okorokov, V. V. Runov, B. P. Toperverg, A. D. Tretyakov, E. I. Maltsev, I. M. Puzeii, and V. E. Mikhailova, Pis'ma Zh. Eksp. Teor. Fiz. 43, 390 (1986) [JETP Lett. 43, 503 (1986)].

[18] V. Deriglazov, A. Okorokov, V. Runov, B. Toperverg, R. Kampmann, H. Eckerlebe, W. Schmidt, and W. Lobner, Physica B 262, 180 (1992).

[19] B. P. Toperverg, V. V. Deriglazov, and V. E. Mikhailova, Physica B 183, 326 (1993). 
[20] S. V. Grigoriev, S. V. Maleyev, V. V. Deriglazov, A. I. Okorokov, N. H. van Dijk, E. Bruck, J. C. P. Klaasse, H. Eckerlebe, and G. Kozik, Appl. Phys. A 74, s719 (2002).

[21] S. V. Grigoriev, E. V. Altynbayev, H. Eckerlebe, and A. I. Okorokov, J. Surf. Invest.: X-Ray, Synchrotron Neutron Tech. 8, 1027 (2014).

[22] M. Kataoka, J. Phys. Soc. Jpn. 56, 3635 (1987).

[23] S. V. Grigoriev, S.-A. Siegfried, E. V. Altynbayev, N. M. Potapova, V. Dyadkin, E. V. Moskvin, D. Menzel, A. Heinemann, S. N. Axenov, L. N. Fomicheva, and A. V. Tsvyashchenko, Phys. Rev. B 90, 174414 (2014).

[24] A. Tsvyashchenko, J. Less-Common Met. 99, L9 (1984).
[25] S. M. Stishov, A. E. Petrova, S. Khasanov, G. K. Panova, A.A. Shikov, J. C. Lashley, D. Wu, and T. A. Lograsso, Phys. Rev. B 76, 052405 (2007).

[26] S. M. Stishov et al., J. Phys.: Condens. Matter 20, 235222 (2008).

[27] A. E. Petrova and S. M. Stishov, J. Phys.: Condens. Matter 21, 196001 (2009).

[28] M. Janoschek, M. Garst, A. Bauer, P. Krautscheid, R. Georgii, P. Böni, and C. Pfleiderer, Phys. Rev. B 87, 134407 (2013).

[29] H. Wilhelm, A. O. Leonov, U. K. Rößler, P. Burger, F. Hardy, C. Meingast, M. E. Gruner, W. Schnelle, M. Schmidt, and M. Baenitz, Phys. Rev. B 94, 144424 (2016). 